\title{
Spectra of negative particles in Au+Au collisions from BES-I at RHIC and $z$-scaling
}

\author{
Mikhail Tokarev ${ }^{1, *}$, Armen Kechechyan ${ }^{1, * *}$, and Imrich Zborovsky ${ }^{2, * * *}$ \\ ${ }^{1}$ Joint Institute for Nuclear Research, 141980 Dubna, Russia \\ ${ }^{2}$ Nuclear Physics Institute of the CAS, 25068 Řež, Czech Republic
}

\begin{abstract}
Negative particle yields in $A u+A u$ collisions obtained by the STAR Collaboration from BES-I at RHIC are analyzed in the framework of the $z$ scaling approach. The spectra were measured over a wide range of collision energy $\sqrt{s_{N N}}=7.7-200 \mathrm{GeV}$ and transverse momentum of produced particles for different centralities at $|\eta|<0.5$. The scaling behavior of the spectra as a function of the collision energy and centrality in z-presentation is verified. The constituent energy loss in dependence on the energy and centrality of collision and transverse momentum of inclusive particle is estimated. New indication on self-similarity of fractal structure of nuclei and fragmentation process is found. A discontinuity of the model parameters - fractal and fragmentation dimensions and specific heat - is considered from the point of view of a phase transition in nuclear matter.
\end{abstract}

\section{Introduction}

Experimental results obtained at RHIC support the hypothesis that a strongly-coupled nuclear medium with parton degrees of freedom, namely the Quark-Gluon Plasma (QGP), is created in heavy-ion collisions in the high energy region $\sqrt{s_{N N}}=62-200 \mathrm{GeV}$ [1-3]. Among the properties of the new medium there is opacity characterized by the suppression of particle yields and viscosity which is found to be such small that the matter looks like an ideal liquid rather than a gas of free quarks and gluons. The determination of features of the phase diagram of nuclear matter created at various temperatures and energy densities is one of the main goals of investigation performed in the heavy ion program at RHIC. Among other tasks there is search for the existence and location of the phase boundaries and a critical point (CP) of the QCD matter. It is considered that systems located nearby the boundary or CP should reveal a discontinuity, enhancement and increased fluctuations and correlations in some of their characteristics. However, there were found no clear indications of such a behavior in the matter produced in nuclear collisions up to now. In order to resolve the problem, the systematic study known as the Beam Energy Scan (BES) program of particle production in $A u+A u$ collisions over a wide range of centrality and energy was suggested. The first phase of the program has been successfully performed (see [4] and references therein).

\footnotetext{
*e-mail: tokarev@jinr.ru

**e-mail: kechechyan@jinr.ru

***e-mail: zborovsky@ujf.cas.cz
} 
In the paper we present new results of analysis of the BES-I STAR data on spectra of negative hadrons produced in $A u+A u$ collisions [5]. The analysis was performed in the framework of the $z$-scaling approach $[6,7]$. The scaling properties of the data $z$-presentation and the characteristics of the constituent subprocess (momentum fractions, recoil mass and energy loss) are studied in dependence on the energy and centrality of collisions.

\section{Negative particle spectra from STAR BES-I}

The transverse momentum distributions of hadrons produced in the high-energy collisions of heavy ions reflect features of the constituent interactions in nuclear medium. The medium modification (energy loss, recombination, coalescence, multiple scattering,...) affects the shapes of the spectra. This allows to study properties of the created matter by variation of the event centrality and collision energy. The STAR Collaboration measured $p_{T}$ spectra of charged hadrons [5] at the energies $\sqrt{s_{N N}}=7.7-200 \mathrm{GeV}$ in the mid-pseudorapidity $|\eta|<0.5$ for the centrality classes of $(0-5) \%,(5-10) \%,(10-20) \%,(20-40) \%,(40-60) \%,(60-80) \%$ over a wide momentum range $0.2<p_{T}<12 \mathrm{GeV} / \mathrm{c}$.

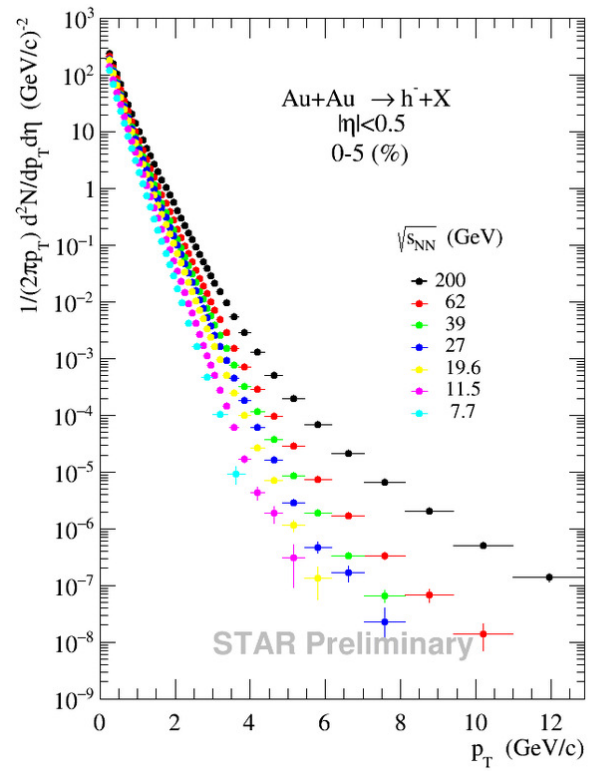

a)

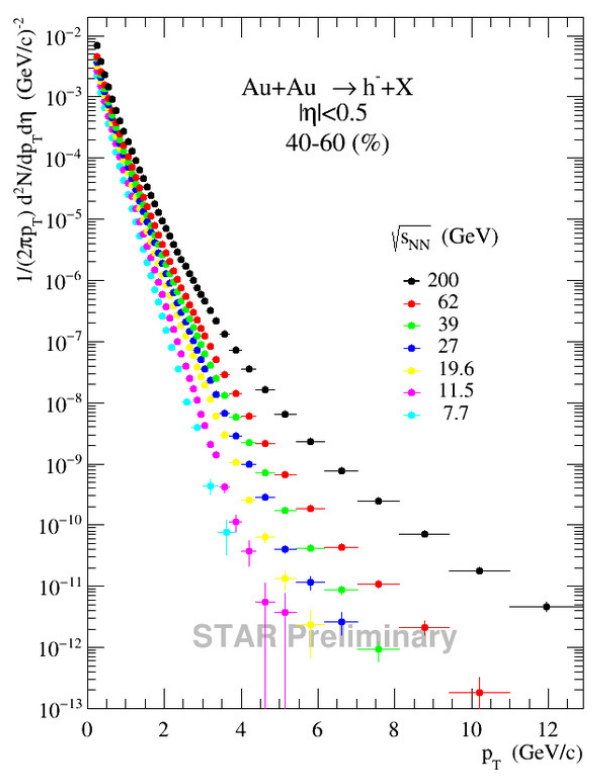

b)

Figure 1. Inclusive spectra of negative hadrons produced in the most central (a) and peripheral (b) $A u+A u$ collisions at $\sqrt{s_{N N}}=7.7-200 \mathrm{GeV}$ and $|\eta|<0.5$. The symbols correspond to the experimental data obtained by the STAR Collaboration at RHIC [5]. Error bars shown are statistical only

Fig. 1 shows the transverse momentum spectra at the energies $\sqrt{s_{N N}}=$ 7.7, 11.5, 19.6, 27, 62.4 and $200 \mathrm{GeV}$ in the mid-pseudorapidity $|\eta|<0.5$ for the most central $(0 \%-5 \%)$ and the peripheral $(40 \%-60 \%)$ collisions. The errors shown are statistical only. The spectra demonstrate the exponential and the power behavior in dependence of $p_{T}$ at low and high $\sqrt{s_{N N}}$, respectively. As a function of the collision energy, the spectra show different sensitivity to the momentum $p_{T}$ for the most central and peripheral collisions, especially in the high- $p_{T}$ range. One can see that, for the transverse momentum $p_{T}=4 \mathrm{GeV} / \mathrm{c}$, the dif- 
ference between the spectra at the energy $\sqrt{s_{N N}}=7.7$ and $200 \mathrm{GeV}$ is about two orders of magnitude.

\section{$3 z$-Scaling approach}

Here we briefly remind the basic ideas of the $z$-scaling approach [6, 7]. It is assumed that the collision of two hadrons or nuclei at high energies can be considered as an ensemble of individual interactions of their constituents. The gross features of an inclusive cross section of a single particle can be expressed in terms of the kinematic characteristics of the corresponding constituent subprocess. The elementary subprocess is considered as a binary collision of the constituents with masses $\left(x_{1} M_{1}\right)$ and $\left(x_{2} M_{2}\right)$ which result in the scattered and recoil objects in the final state with masses $\left(m_{a} / y_{a}\right)$ and $\left(x_{1} M_{1}+x_{2} M_{2}+m_{b} / y_{b}\right)$, respectively. The constituents of the incoming hadrons (or nuclei) with masses $M_{1}, M_{2}$ and momenta $P_{1}, P_{2}$ carry their fractions $x_{1}, x_{2}$. The produced secondary objects transform into real particles after the constituent collisions. The registered particle with mass $m_{a}$ and the 4-momentum $p$ carries the fraction $y_{a}$ of the 4-momentum of the scattered constituent. Its hadron counterpart with mass $m_{b}$, moving in the opposite direction, carries the 4-momentum fraction $y_{b}$ of the produced recoil. The momentum conservation law in the constituent subprocess is connected with the recoil mass $M_{X}=\left(x_{1} M_{1}+x_{2} M_{2}+m_{b} / y_{b}\right)$ of the recoil object as follows

$$
\left(x_{1} P_{1}+x_{2} P_{2}-p / y_{a}\right)^{2}=M_{X}^{2} .
$$

The associated production of the particle with the mass $m_{b}$ ensures conservation of the additive quantum numbers (the electric charge, baryon number, strangeness, charm, beauty). Eq. (1) is an expression of the locality of hadron interaction at a constituent level. It poses a constraint on the momentum fractions $x_{1}, x_{2}, y_{a}$, and $y_{b}$ which determine the constituent subprocess.

The structure of the colliding objects and fragmentation of the systems moving in the scattered and recoil directions are characterized by the structural parameters $\delta_{1}, \delta_{2}$, and $\epsilon_{a}, \epsilon_{b}$, respectively. We connect the parameters with the corresponding momentum fractions $x_{1}, x_{2}$, $y_{a}$ and $y_{b}$ by the function

$$
\Omega=\left(1-x_{1}\right)^{\delta_{1}}\left(1-x_{2}\right)^{\delta_{2}}\left(1-y_{a}\right)^{\epsilon_{a}}\left(1-y_{b}\right)^{\epsilon_{b}} .
$$

Physical interpretation of $\Omega$ is given by its proportionality to relative number of all such constituent configurations in the inclusive reaction which contain the configuration defined by the fractions $x_{1}, x_{2}, y_{a}$, and $y_{b}$. The function $\Omega$ plays the role of a relative volume which occupy these configurations in space of the momentum fractions. The structural parameters $\delta_{1}, \delta_{2}$, $\epsilon_{a}$ and $\epsilon_{b}$ are interpreted as fractal dimensions in the corresponding space of the momentum fractions. For the unpolarized proton-proton collisions we have $\delta_{1}=\delta_{2} \equiv \delta$. In the case of nucleus-nucleus collisions there are relations $\delta_{1}=A_{1} \delta$ and $\delta_{2}=A_{2} \delta$, where $A_{1}, A_{2}$ are atomic numbers. We assume that objects produced in constituent subprocesses in the scattered and recoil directions have equal fragmentation properties which can be described by the same parameter $\epsilon$. We set therefore $\epsilon_{a}=\epsilon_{b} \equiv \epsilon_{F}$, where the subscript $(F)$ denotes dependence on type of the inclusive particle.

For given values of $\delta$ and $\epsilon_{F}$, the fractions $x_{1}, x_{2}, y_{a}$ and $y_{b}$ are determined in a way to maximize the function $\Omega\left(x_{1}, x_{2}, y_{a}, y_{b}\right)$, simultaneously fulfilling condition (1). This maximal $\Omega$ is used in the definition (3) of the scaling variable $z$. The parameter $\epsilon_{F}$ enables to take effectively into account also prompt resonances out of which the inclusive particle of a given type may be created. At fixed masses $m_{a}$ and $m_{b}$, larger values of $\epsilon_{F}$ correspond to smaller $y_{a}$ 
and $y_{b}$, which in turn give larger ratios $m_{a} / y_{a}$ and $m_{b} / y_{b}$. In our phenomenological approach this means that production of the inclusive particle with mass $m_{a}$ and its counterpart with mass $m_{b}$ is a result of fragmentation from larger masses which mimic in a sense processes with prompt resonances.

The self-similarity of hadron interactions reflects a property that hadron constituents and their interactions are similar. The self-similarity parameter $z$ is defined as follows

$$
z=z_{0} \Omega^{-1}
$$

where $\Omega$ is maximal value of (2) with condition (1) and $z_{0}=\sqrt{s_{\perp}} /\left[\left(d N_{c h} /\left.d \eta\right|_{0}\right)^{c} m_{N}\right]$. The quantity $z$ is proportional to the transverse kinetic energy $\sqrt{s_{\perp}}$ of the constituent subprocess consumed on the production of the inclusive particle $\left(m_{a}\right)$ and its counterpart $\left(m_{b}\right)$. The symbol $m_{N}$ stands for the nucleon mass. The quantity $d N_{c h} /\left.d \eta\right|_{0}$ is the corresponding multiplicity density of charged particles produced in the central region of the inclusive reaction at pseudorapidity $\eta=0$. The value of $d N_{c h} /\left.d \eta\right|_{0}$ is raised to the power of $c$. The multiplicity density in the central interaction region is related to a state of the produced medium. The parameter $c$ characterizes properties of this medium [7]. It is determined from multiplicity dependence of inclusive spectra. The variable $z$ is expressed via the momentum fractions $\left(x_{1}, x_{2}, y_{a}, y_{b}\right)$, multiplicity density, and the parameters $\delta_{1}, \delta_{2}, \epsilon_{a}, \epsilon_{b}$ and $c$.

The scaling function $\psi(z)$ is expressed in terms of the experimentally measured inclusive invariant cross section $E d^{3} \sigma / d p^{3}$, the multiplicity density $d N / d \eta$ and the total inelastic cross section $\sigma_{\text {in }}$ as follows

$$
\psi(z)=\frac{\pi}{(d N / d \eta) \sigma_{\text {inel }}} J^{-1} E \frac{d^{3} \sigma}{d p^{3}},
$$

where $J$ is Jacobian for the transformation from $\left\{p_{T}^{2}, y\right\}$ to $\{z, \eta\}$. The Jacobian is a function of kinematic variables characterizing the inclusive process. The multiplicity density $d N / d \eta$ in the expression (4) concerns particular hadrons species. It depends on the center-of-mass energy and the production angles at which the inclusive spectra were measured. The function $\psi(z)$ is normalized as follows

$$
\int_{0}^{\infty} \psi(z) d z=1
$$

The relation allows us to interpret $\psi(z)$ as a probability density to produce an inclusive particle with the corresponding value of the self-similar variable $z$.

\section{Self-similarity in Au-Au collisions}

The idea of the BES programs at RHIC is to span the phase diagram of QCD matter from the top RHIC energy to the lowest possible energy [4]. The aim is to search for the phase boundary between confined and unconfined phases and location of a CP.

It is known $[8,9]$ that study of the critical phenomena is most preferable in terms of dimensionless variables. The use of such variables for a description of the phenomena related to self-similarity of hadron interactions appears to be a useful concept. Important manifestation of this concept is a notion of the scaling itself. The scaling in general means a self-similarity at different scales. The approach based on the $z$-scaling suggested in $[6,7]$ is treated as a manifestation of the self-similarity of structure of the colliding objects (hadrons, nuclei), the interaction mechanism of their constituents, and the process of fragmentation into real hadrons in the final state. We use the approach for the analysis of spectra of charged hadrons obtained by the STAR Collaboration in the BES-I program [4] at RHIC.

The $z$-presentation of the spectra of negative hadrons [5] measured by the STAR Collaboration in $A u+A u$ collisions at $\sqrt{s_{N N}}=7.7$ and $39 \mathrm{GeV}$ in the pseudorapidity window $|\eta|<0.5$ 
is illustrated in Fig.2. The different symbols correspond to the transverse momentum distributions for different centrality classes of the nuclei collisions. One can see a "collapse" of the symbols representing the STAR data onto a single curve. The scaling function $\psi(z)$ changes more than ten orders of magnitude. The self-similarity variable $z$ varies in the range $z=0.1-30$. We have checked that the scaling is valid for all centralities and collision energies in the range $\sqrt{s_{N N}}=7.7-200 \mathrm{GeV}$.

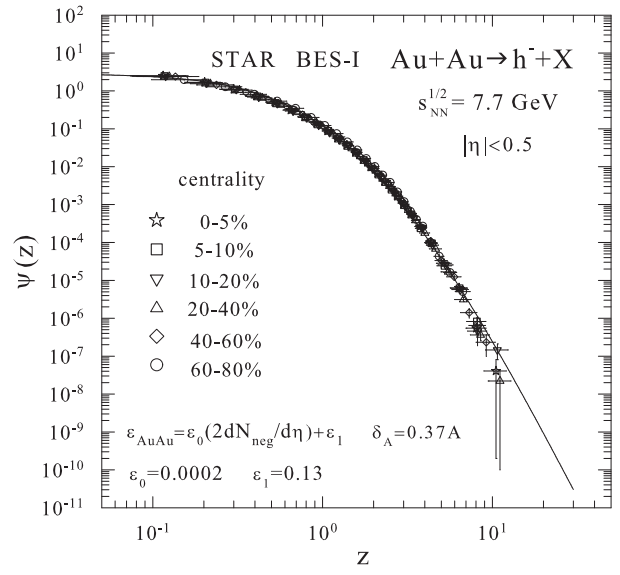

a)

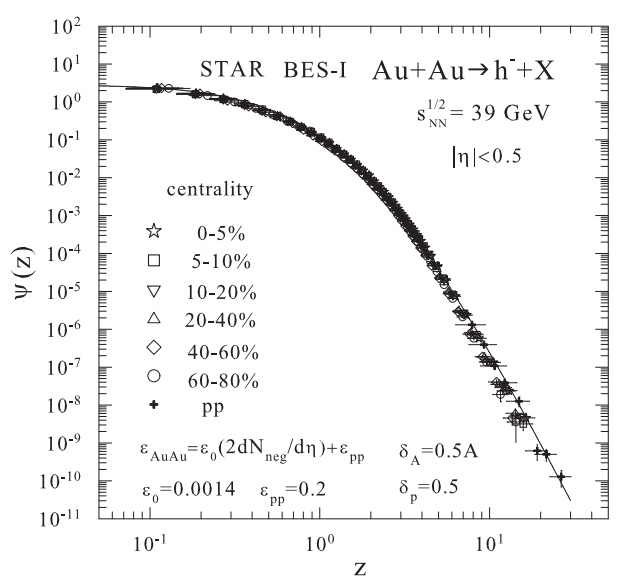

b)

Figure 2. Inclusive spectra of negative hadrons produced in $A u+A u$ collisions at $\sqrt{s_{N N}}=7.7$ (a) and $39 \mathrm{GeV}$ (b) for different centrality classes and $|\eta|<0.5$ shown in $z$-presentation. The symbols correspond to the experimental data obtained by the STAR Collaboration at RHIC [5]. The solid line is a reference curve for $p+p$ collisions

The solid curve depicts the $z$-scaling of $h^{-}$particles produced in $p+p$ interactions over the range $\sqrt{s}=11.5-200 \mathrm{GeV}, p_{T}=0.2-13 \mathrm{GeV} / \mathrm{c}$ and $\theta_{c m s}=90^{\circ}$. It corresponds to the parameters $\delta_{p}=0.5, c_{p p}=0.25$ and $\epsilon_{p p}=0.2$. Fig. 2 demonstrates approximate matching of the symbols and the solid curve provided the fractal dimension $\epsilon_{A A}$ of the fragmentation processes in the nuclear system depends linearly on the multiplicity density characterizing different centrality classes of the nuclei collisions. It was found that the transverse momentum spectra of negative hadrons produced in $A u+A u$ collisions demonstrate energy independence of $\psi(z)$ over the analyzed kinematic range at constant values of the parameters $\delta_{A}=A \delta_{p}$, $c_{A A}=0.11$ and $\epsilon_{p p}=0.2$ for $\sqrt{s_{N N}}>19.6 \mathrm{GeV}$.

\section{Constituent subprocess}

The momentum fractions $x_{1}$ and $x_{2}$ obtained from maximization of $\Omega$ with the condition (1) determine the energy of the underlying constituent subprocess. The fractions are equal to each other $\left(x_{1}=x_{2}\right)$ for particles detected at the angle $\theta_{c m s}=90^{\circ}$ in the center-of-mass system of $A u+A u$ collisions.

Fig. 3 shows the dependence of $A x_{1}$ on the transverse momentum $p_{T}$ of $h^{-}$hadrons produced in $A u+A u$ interactions at $\sqrt{s_{N N}}=7.7$ and $39 \mathrm{GeV}$ in the pseudorapidity window $|\eta|<0.5$ for different centralities. The momentum fraction $A x_{1}$ increases with $p_{T}$ and decreases with the increasing collision energy. We observe larger sensitivity of the fraction to the centrality of collisions for higher $\sqrt{s_{N N}}$. The kinematic limit of the inclusive reaction is defined by the condition $A x_{1}=A x_{2}=A$. The regime $A x_{1}, A x_{2} \rightarrow A$ corresponds to the configurations when almost all of the collision energy is carried by single constituents, which 
interact via the underlying subprocesses. An extremely strong cumulation of nuclei (compression of hadron matter) takes place in this case. According to the ideas of hadron structure at small scales envisaged in the $z$-scaling scheme, this would correspond to coherent multiple interactions of partons trapped in the constituents which carry the large fractions of momenta of the colliding nuclei. For the analysed STAR data, the fraction $A x_{1}$ is larger than unity only at the energy $\sqrt{s_{N N}}=7.7$ and $11.5 \mathrm{GeV}$ in the high $p_{T}$ tail of the spectra. The value of $A x_{1}=1$ corresponds to the kinematic limit for $p+p$ reaction.

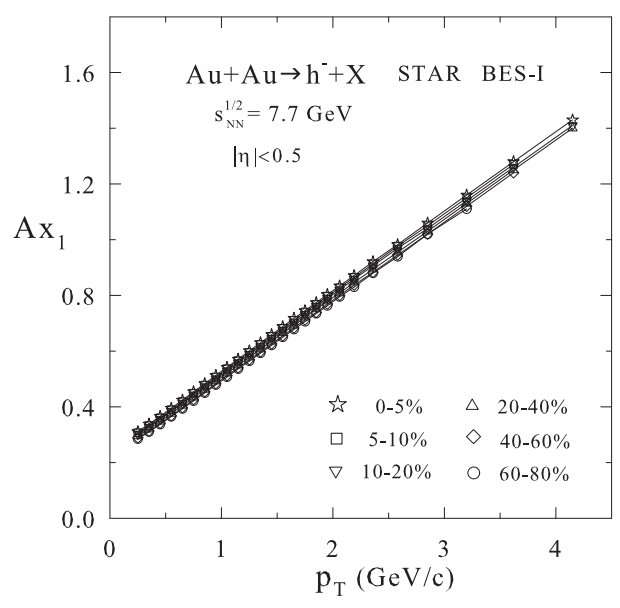

a)

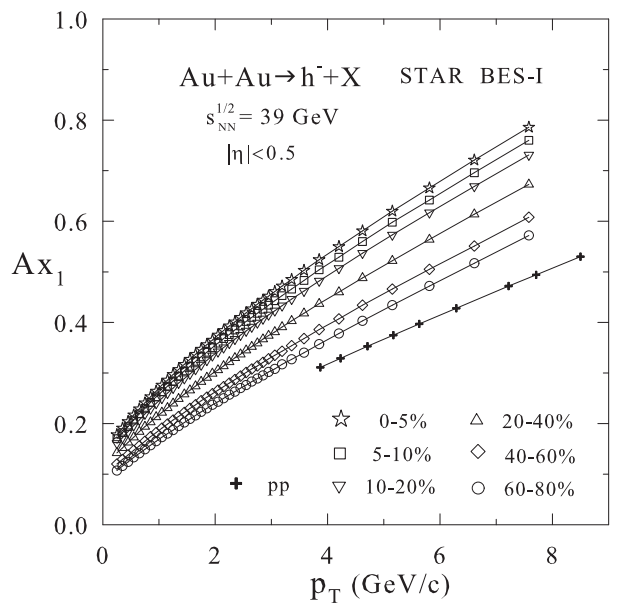

b)

Figure 3. The dependence of the momentum fraction $A x_{1}$ on the transverse momentum $p_{T}$ of $h^{-}$ hadrons produced in $A u+A u$ collisions at the energy $\sqrt{s_{N N}}=7.7$ (a) and $39 \mathrm{GeV}$ (b) for different centralities and $|\eta|<0.5$

The momentum fraction $y_{a}$ is related with amount of the constituent energy loss and its dependence on the collision energy and transverse momentum of the produced inclusive particle. It has relevance to the evolution of the matter created in proton-proton or nucleusnucleus collisions. The quantity that has an immediate relation to the processes with energy losses is the fractal dimension $\epsilon_{A A}$ in the $z$-scaling approach. Its value influences mostly the momentum fraction $y_{a}$. The relative energy loss of the scattered constituent with energy $E_{q}$ is given by the relation $\Delta E_{q} / E_{q}=\left(1-y_{a}\right)$. Its dependence on the energy $\sqrt{s_{N N}}$, the multiplicity density of charged particles $d N_{c h}^{A A} / d \eta$ and the momentum $p_{T}$ of the inclusive particle gives information on the medium created in the nuclear collisions and also on properties of the fragmentation processes in the produced matter.

Fig. 4 shows the fraction $y_{a}$ as a function of the transverse momentum $p_{T}$ of $h^{-}$hadron produced in $A u+A u$ collisions at $\sqrt{s_{N N}}=7.7$ and $39 \mathrm{GeV}$ at mid-pseudorapidity $|\eta|<0.5$ for different centrality classes. The points demonstrate a non-linear monotonic growth of $y_{a}$ with $p_{T}$. This means that the relative energy dissipation associated with a high- $p_{T}$ particle is smaller than for the inclusive processes with lower transverse momenta. A decrease of $y_{a}$ with the increasing collision energy corresponds to more energy dissipation at higher energies when the charged particle multiplicity is higher. As seen from Fig.4, the fraction $y_{a}$ decreases at a fixed collision energy and for a fixed momentum $p_{T}$ of the produced particle, as the centrality of collision becomes larger. It means that the more dense nuclear medium dissipates larger amount of energy. A stronger sensitivity of $y_{a}$ to the centrality of collisions is found at the higher energy $\sqrt{s_{N N}}=39 \mathrm{GeV}$ in comparison to the sensitivity of $y_{a}$ at the lower energy $\sqrt{s_{N N}}=7.7 \mathrm{GeV}$. The points corresponding to $y_{a}$ for $p+p$ collisions are located above the 


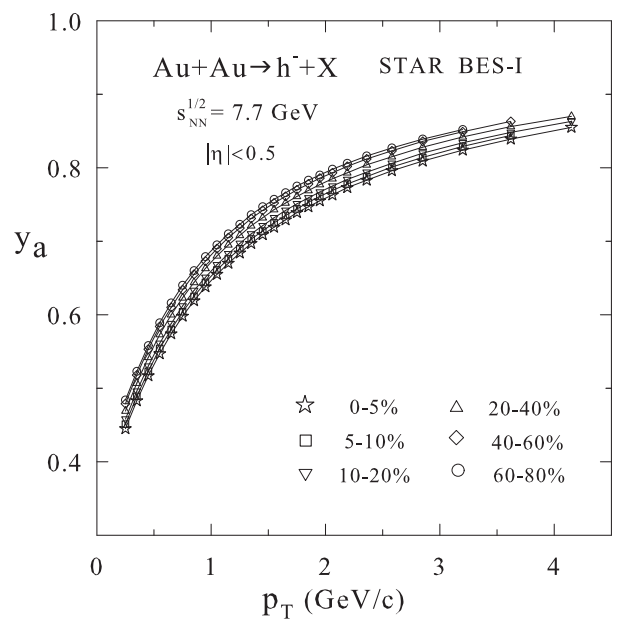

a)

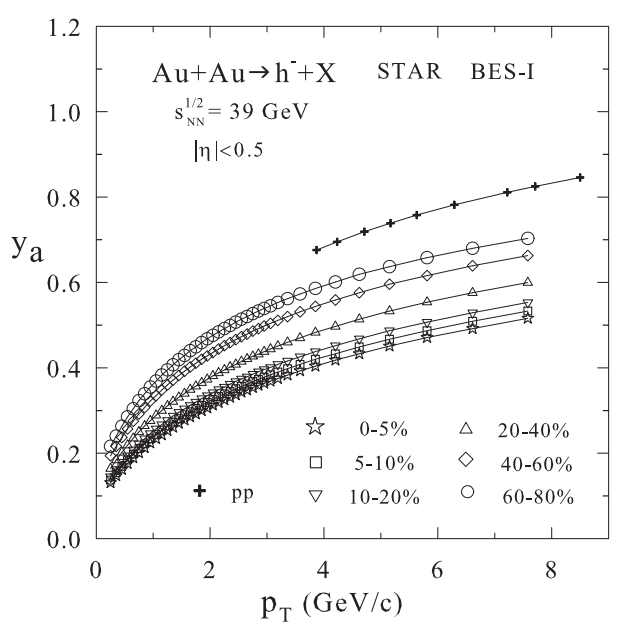

b)

Figure 4. The dependence of the fraction $y_{a}$ on the transverse momentum $p_{T}$ of $h^{-}$hadron produced in $A u+A u$ collisions at the energy $\sqrt{s_{N N}}=7.7$ (a) and $39 \mathrm{GeV}$ (b) for different centralities and $|\eta|<0.5$.

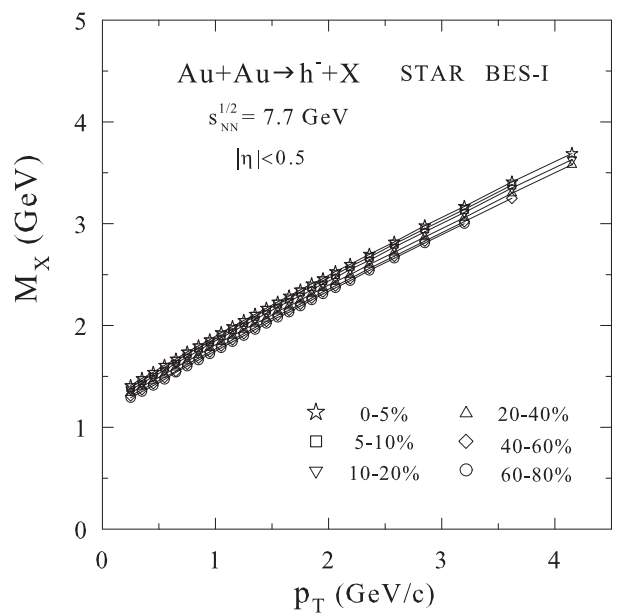

a)

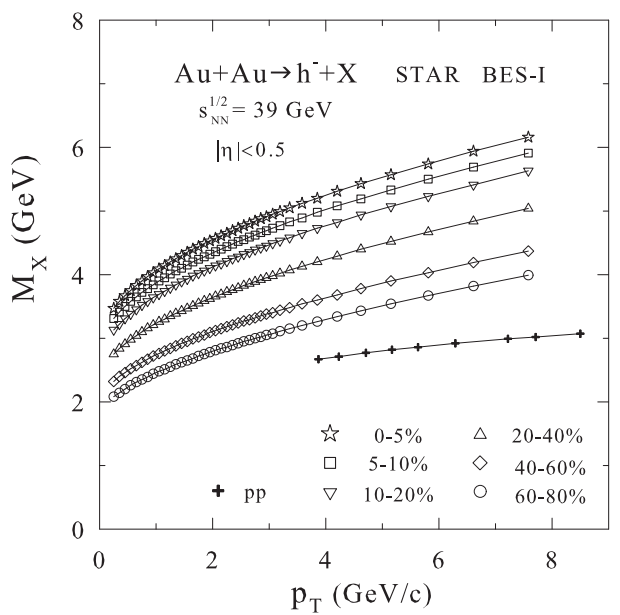

b)

Figure 5. The dependence of the recoil mass $M_{X}$ on the transverse momentum $p_{T}$ of $h^{-}$hadron produced in $A u+A u$ collisions at the energy $\sqrt{s_{N N}}=7.7$ (a) and $39 \mathrm{GeV}$ (b) for different centralities and $|\eta|<0.5$.

points for the $A u+A u$ collisions for all $p_{T}$. It means that the energy loss is minimal in the absence of nuclei.

The recoil object in the constituent subprocess moving in the away side direction of the inclusive particle is characterized by the mass $M_{X}\left(x_{1}, x_{2}, y_{b}\right)=x_{1} M_{1}+x_{2} M_{2}+m_{b} / y_{b}$. The mass $M_{X}$ has internal connection to the structure of the colliding objects, constituent interactions, and fragmentation processes which lead to the production of the individual hadrons. The part of the recoil mass proportional to $m_{b}$ is dominant for small values of $y_{b}$. Its $p_{T}$-dependence is governed by the values of the fragmentation dimension $\epsilon_{A A}$. 
Fig. 5 shows the dependence of the recoil mass $M_{X}$ on the transverse momentum of negative particles produced in $A u+A u$ collisions at the energy $\sqrt{s_{N N}}=7.7$ and $39 \mathrm{GeV}$ for different centralities (0-5)\% - (60-80)\% in the central pseudorapidity region $|\eta|<0.5$. As one can see, the recoil mass increases smoothly with $p_{T}$ and $\sqrt{s_{N N}}$. The centrality dependence, clearly seen at $\sqrt{s_{N N}}=39 \mathrm{GeV}$, diminishes with the decreasing energy and at the energy $\sqrt{s_{N N}}=7.7 \mathrm{GeV}$, the recoil mass $M_{X}$ practically does not depend on the centrality of collisions. The $p_{T}$-dependence of $M_{X}$ is influenced mainly by the momentum fraction $y_{b}$, which is much smaller than $y_{a}$. Relatively small values of $y_{b}$ mean that the momentum balance in the production of an inclusive particle is more likely compensated by many particles with smaller momenta than by a single particle with higher momentum moving in the opposite direction. Therefore the growth of $M_{X}$ with $\sqrt{s_{N N}}$ corresponds to an evolution of the system as its entropy increases.

\section{Conclusions}

Results of a new analysis in the framework of the $z$-scaling approach of the negative particle spectra obtained by the STAR Collaboration in $A u+A u$ collisions in the first phase of the RHIC BES program were presented. The measured spectra cover a wide range of collision energy $\sqrt{s_{N N}}=7.7-200 \mathrm{GeV}$ and transverse momentum $p_{T}=0.2-12 \mathrm{GeV} / \mathrm{c}$ of produced particles at different centralities in the pseudorapidity range $|\eta|<0.5$. The scaling behavior of the spectra in $z$-presentation as a function of the collision energy and centrality was verified. The results presented for $A u+A u$ collisions support the properties of data $z$-presentation found in our previous analyses of the inclusive spectra of different hadrons produced in proton-proton collisions, which have been measured at the accelerators ISR, SPS, Tevatron and RHIC.

The $z$-scaling approach was developed as a method of data analysis based on the fundamental symmetry principles such as the self-similarity, locality, and fractality of hadron interactions at high energies. The approach was applied here for a description of hadron production in heavy ion collisions. The scaling function $\psi(z)$ and the self-similarity parameter $z$ are expressed via measurable quantities and some model parameters which allow physical interpretation. The scaling variable $z$ depends on three model parameters $\delta_{A}, \epsilon_{A A}$ and $c_{A A}$. The parameters $\delta_{A}$ and $\epsilon_{A A}$ characterize fractal structure of the colliding nuclei and fragmentation processes in the final state, respectively. The third parameter, $c_{A A}$, is interpreted as a "specific heat" of the produced medium. It was found that the $z$-scaling is consistent with $c_{A u A u}=0.11$ and $\delta_{A}=0.5 A$. The fragmentation dimension $\epsilon_{A A}$ increases with multiplicity density. The scaling function $\psi(z)$ reveals universality of shape and manifests two regimes of behavior the saturation at low- $z$ and the power law at high- $z$ range.

Some properties of the constituent processes were studied. A monotonic growth of the momentum fractions $A x_{1}$ and $y_{a}$ with $p_{T}$ was found for all collision energies and all centralities. The constituent energy loss as a function of energy and centrality of collisions and transverse momentum of inclusive particle was estimated. The presented results support a microscopic scenario of hadron production as formation of fractal-like objects in the medium created in heavy ion collisions. The results extend the applicability of the self-similarity and the fractality principle to the description of hadron production in nucleus-nucleus collisions. Further detailed study is needed to search for a discontinuity of the model parameters - the fractal and fragmentation dimensions and "specific heat", which is assumed as a signature of critical phenomena (phase transition, CP) in nuclear matter. Based on the obtained results we conclude that new confirmation of self-similarity of negative hadron production in heavy ion collisions at RHIC energies was found. 
Acknowledgments. This work was partially supported by Project funded by the MEYS of the Czech Republic under the contract LTT18021.

\section{References}

[1] B. I. Abelev et al. (STAR Collaboration), Experimental Study of the QCD Phase Diagram $\mathcal{E}$ Search for the Critical Point: Selected Arguments for the Run-10 Beam Energy Scan (June 4, 2009) http://drupal.star.bnl.gov/STAR/starnotes/public/sn0493

[2] M. M. Aggarwal et al. (STAR Collaboration), An Experimental Exploration of the QCD Phase Diagram: The Search for the Critical Point and the Onset of Deconfinement, arXiv: 1007.2613

[3] STAR Collaboration, Studying the Phase Diagram of QCD Matter at RHIC. A STAR white paper summarizing the current understanding and describing future plans, SN0598, (June 1, 2014)

[4] L. Adamczyk et al. (STAR Collaboration), Phys. Rev. C 96, 044904 (2017)

[5] M. V. Tokarev (for the STAR Collaboration), Int. J. Mod. Phys. Conf. Ser. 39, 1560103 (2015)

[6] I. Zborovský, M. V. Tokarev, Phys. Rev. D 75, 094008 (2007)

[7] I. Zborovský, M. V. Tokarev, Int. J. Mod. Phys. A 24, 1417 (2009)

[8] H. E. Stanley, Introduction to Phase Transitions and Critical Phenomena (Oxford University Press, Oxford, 1971) 333

[9] H. E. Stanley, Rev. Mod. Phys. 71, S358 (1999) 